\title{
Statistical programming: small mistake, big impact
}

Coding mistakes can lead to false results. Statisticians and data scientists should exploit best practices and tools in statistical programming to enhance reproducible analyses. By Simon Schwab and Leonhard Held.

[Keep main text to 750-1000 words]

The analysis of a 2019 randomized controlled trial showed a treatment benefit for patients with obstructive pulmonary disease (COPD). The intervention was a 3-month self-management program delivered by COPD-specialized nurses, the control group was usual care. The study found that the primary outcome, the mean number of hospitalizations or emergency department visits, was significantly lower in the treatment group (difference in means $-0.68,95 \%-\mathrm{Cl}$ from -1.15 to $-0.22, p=0.004$ ). So far, so good.

Ten months later the article was retracted. What happened? The study authors detected a programming error in the analysis of the dataset. The group assignment (treatment vs. control) was coded in the format of " 1,2 " and was converted to a binary type of " 0 , 1", but the assignment was made incorrectly and resulted in a reversed coding of the study groups [1]. Thus, the correct result reversed the main finding. There were on average 0.68 more COPD-related hospitalizations and emergency department visits in the intervention group (95\%-Cl from 0.22 to 1.15$)$. So there was unexpected harm from the treatment. Sometimes a small mistake can have a big impact.

A treatment effect estimate from a clinical study is just the tip of the iceberg [2]. Data analysis is a process with many stages that starts with data collection, data cleaning, data analysis and statistical inference. Mistakes can happen at all stages. After the authors of the COPD study detected the error and reanalyzed the data, another mistake was found in the imputation of missing values of the co-primary outcome.

This example underlines the importance of the data scientist in collaborative research projects, sadly its role is often undervalued. Statistical training is key, but advanced programming skills, complete documentation with computational notebooks, and best practices from software engineering are crucial to avoid statistical programming mistakes. Table 1 is an incomplete list of avoidable statistical programming practices that can lead to mistakes. Statisticians who develop statistical software packages are already familiar with these recommendations, as well as researchers with a background in computer science.

\section{Programming skills}

Programming skills are a large plus when it comes to data analysis. Researchers are well devised to take courses in programming at the university level. Another option are the Carpentries (carpentries.org), a non-profit organization that teaches computer programming and data science skills to researchers. Python and $\mathrm{R}$ are becoming the favorite tools among data scientists, but these are programming languages in the first place. The sheer amount of data today, the need to combine datasets, and data cleaning is hard to imagine without proficient programming skills. 
Such skills include knowledge about different data types, boolean expressions, conditionals and loops, string manipulations and writing functions or packages [3].

Mistakes can be more easily detected when code and data are openly shared with a research publication. Importantly, the code needs to be readable and should not be messy. This can be achieved by following naming conventions and writing comments when necessary to explain the code. After reanalysing and republishing the COPD study the authors could have released a minimal dataset together with a computational notebook to reproduce the main finding of the study. Some journals already request authors' statistical code to promote reproducible research [4].

In clinical research data are often not available due to potential identifiable information. Requesting data access is associated with considerable effort, but ClinicalStudyDataRequest.com (CSDR) aims to facilitate access to patient-level data. Practical guidance on anonymising trial datasets are available, but need to balance anonymity with data utilization [5]

\section{Dynamic reporting with computational notebooks}

Computational notebooks document all the stages in data reading, data cleaning and transformation, statistical modeling, data visualization, machine learning, and much more in a single electronic document. This document represents a complete computational narrative that discloses all fundamental assumptions and choices on how the final result was obtained which largely facilitate reproducibility. Such notebooks are dynamic reports that embed analysis code, computational output, tables, figures, and explanatory text. When a change is made and or an error is fixed, all results and figures can be regenerated within a few seconds.

Prominent free software to create dynamic reports are knitr for R (yihui.org/knitr), RStudio's RMarkdown for R, Python and SQL (rmarkdown.rstudio.com), and the Jupyter Notebook (jupyter.org) supporting over 40 programming languages. The latter was inspired by the three programming languages Julia (Ju), Python (Py) and R [6]. Computational notebooks can be publicly shared on the internet, ideally with the associated data. Only then the result of a study can be fully reproduced and verified.

\section{Learning from software engineering}

Software developers are the experts when it comes to delivering quality code. They came up with a philosophy called "extreme programming" that includes a set of practices proven useful in improving software development [7]. Among the many principles and practices are, for example, testing and pair programming, see Table 1.

\section{Admit mistakes}

Everyone makes mistakes. The integrity of science is built on the principle that scientists are fully transparent. After the discovery of the errors in the analysis the authors of the COPD study have acknowledged their mistake and took the right steps forward, informed the journal and apologized to the readers. Eventually a new article was published in the same journal with the corrected findings. 
To avoid programming errors the Johns Hopkins Biostatistics Center which was involved in this study, has introduced a new standard operating procedure (SOP) for checking the randomization assignments [1]. SOPs are commonly used in the pharmaceutical industry to ensure good statistical practice and compliance with regulatory requirements, and there are guidelines available to develop SOPs [8]. The new procedure is a verification process to ensure that the group assignment in the analysis matches the assignment provided by the trial team. But even with this SOP in place, the imputation error in the co-primary outcome may have remained undetected.

\section{About the authors}

Simon Schwab is a postdoctoral researcher at the Center for Reproducible Science and the Department of Biostatistics at the University of Zurich (UZH).

Leonhard Held is a professor of biostatistics at $\mathrm{UZH}$ and director of the Center for Reproducible Science at $\mathrm{UZH}$.

\section{References}

1. Aboumatar H, Wise RA. 2019 Notice of Retraction. Aboumatar et al. Effect of a Program Combining Transitional Care and Long-term Self-management Support on Outcomes of Hospitalized Patients With Chronic Obstructive Pulmonary Disease: A Randomized Clinical Trial. JAMA. 2018;320(22):2335-2343. JAMA 322, 1417-1418.

2. Leek JT, Peng RD. 2015 Statistics: $P$ values are just the tip of the iceberg. Nature 520, 612 .

3. Matloff N. 2011 The Art of R Programming: A Tour of Statistical Software Design. 1st edn. No Starch Press.

4. Localio AR, Goodman SN, Meibohm A, Cornell JE, Stack CB, Ross EA, Mulrow CD. 2018 Statistical Code to Support the Scientific Story. Ann. Intern. Med. 168, 828-829.

5. Keerie C, Tuck C, Milne G, Eldridge S, Wright N, Lewis SC. 2018 Data sharing in clinical trials - practical guidance on anonymising trial datasets. Trials 19, 25.

6. Perkel JM. 2018 Why Jupyter is data scientists' computational notebook of choice. Nature 563, 145-146.

7. Beck K, Andres C. 2004 Extreme Programming Explained: Embrace Change. 2nd edn. Addison-Wesley Professional.

8. North PM. 1998 Ensuring Good Statistical Practice in Clinical Research: Guidelines for Standard Operating Procedures (An Update). Drug information journal : DIJ / Drug Information Association 32, 665-682. 
Table 1. Statistical programming practices to avoid.
Programming skills
Arbitrary naming of variables
Naming conventions make the code more readable. The variables ideally are self-explanatory without the need for further commenting, e.g. snakecase or camelcase (passenger_class or passengerClass).
Too many or no
comments
Not using data types efficiently
Writing messy code
Hard coding
Comments are sections in the code that the compiler ignores, which are useful to explain things. If naming conventions are used only few comments are necessary.
There is no reason to code treatment groups as a numerical type $[1,2]$. In $R$ "factors" can be used to code the categorial levels treatment and control.
White space and tabbing are useful to structure and align code. Duplicated code should be avoided and outsourced as functions.
Use constants instead of hard coding of numbers at multiple places directly in the code. Constants are variables that never change and are in full capitals, e.g. SAMPLE_SIZE $=120$.

\section{Computational notebooks}

Using graphical software to obtain results and figures

Inefficient use of packages
Computational notebooks are documents for dynamic reporting that embed code, computations, explanatory text, tables and figures. Such environments include Jupyter Notebooks and $R$ Markdown and improve reproducibility.

Including established packages to delegate responsibility is efficient and more error prone than writing your own code. But including too many packages can also add complexity and unnecessary dependencies.

\section{Learning from software engineering}

Lose old versions of the code

No testing

No code review
Version control tools such as "git" can retain all versions of the code through the lifecycle and also allow collaborative software development when using a centralized service like GitHub (github.com) or GitLab (gitlab.com).

Testing conditions with logical expression using functions like assert ( ) adds confidence in analysis code. It is also a debugging tool that throws an error when a condition is violated, for example, a score that needs to be within a certain range. Package developers use "Unit tests", a set of automated tests to ensure expected behavior. Even obvious things should be tested.

In pair programming two persons work together at one computer. One person writes the code, the other one is the observer who can come up with suggestions. This creates peer-reviewed code that is more error prone and a valuable learning experience as knowledge is constantly shared. 